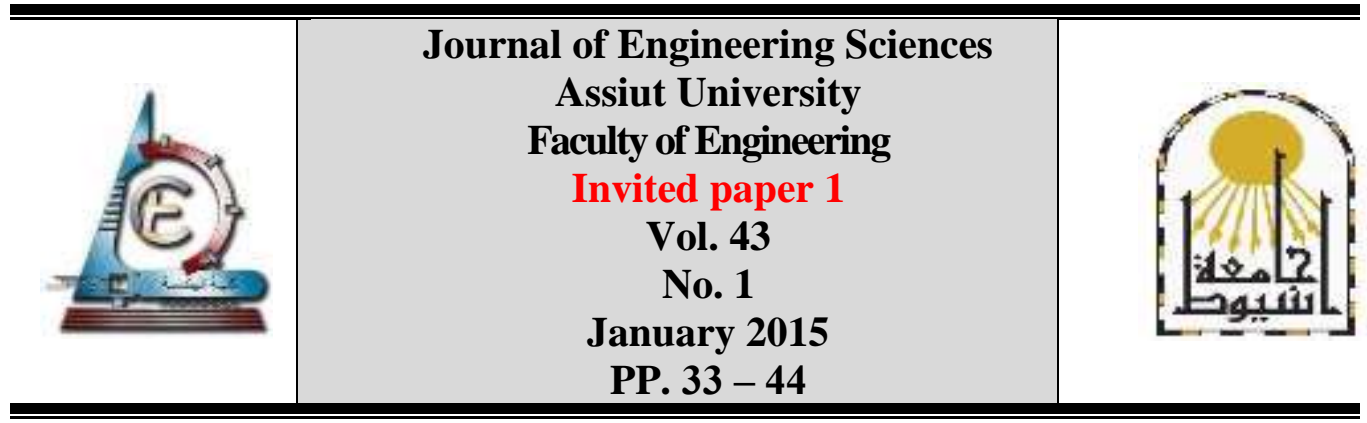

\title{
ROBUST LOAD FREQUENCY CONTROL APPROACH FOR SMART POWER SYSTEMS CONCERNING ELECTRIC VEHICLES AND HEAT PUMP WATER HEATERS
}

\author{
Yasunori Mitani ${ }^{1}$ and Tarek Hassan Mohamed ${ }^{2}$ \\ ${ }^{1}$ Dept. of Electrical and Electronic Eng., Kyushu Institute of Technology, Kitakyushu, Japan \\ ${ }^{2}$ Faculty of Energy Engineering, Aswan University, Aswan, Egypt
}

\begin{abstract}
In recent years, photovoltaic has generation become the most popular renewable energy based generations. However, this power generation cannot supply constant electric power output and sometimes cause the imbalance between supply and demand. To alleviate the mentioned problem, a number of Heat Pump Water Heaters (HPWH) and Electric Vehicles (EV) are used as new control equipment for Load Frequency Control (LFC) in order to suppress the frequency fluctuation caused by such a large amount of renewable energy sources. However, Due to the system uncertainties such as system parameters variation, the conventional controllers which are designed without taking system uncertainties into account in the controller design may lose the control effect and fail to damp the frequency deviation. This will affect the quality of supply and may deteriorate the system stability. This paper proposes, A New load frequency control (LFC) using the coefficient diagram method (CDM) technique in the presence of variable solar power \& thermal power have been presented. The CDM technique has been designed such that the effect of the uncertainty due to governor and turbine parameters variation and variable solar power \& load disturbance is reduced. Digital simulations for a power system are provided to validate the effectiveness of the proposed scheme. A performance comparison between the proposed and a conventional integral control scheme is carried out confirming the superiority of the proposed CDM technique in smart power system.
\end{abstract}

Keywords: load frequency control; coefficient Diagram method; variable solar power, thermal power, Heat Pump Water Heaters (HPWHs) and Electric Vehicles (EV) and variable load

\section{Introduction}

In recent years, electricity generations by wind power (WP)[1-6] and Photovoltaic (PV)[7-8] have received considerable attention worldwide. The penetration of PV systems in Japan is rising [2]. However, whenever a large number of renewable-power generating stations access a power grid, the necessity of maintaining the power quality of utility- and smart-grid power systems demands that the following issues receive significant consideration and study: 1) stabilization of power quality of islanding/interconnected systems; 2)

\footnotetext{
* Corresponding author.

E mail address: tarekhie@yahoo.com
} 
smoothing of output fluctuation in Photovoltaic (PV) and WP generation; 3) quantifying of economics of new-energy generation; 4) effective integration with intelligent multiuser power system, and; 5) determination of optimal energy generation/storage capacity, among others. Indeed, WP and PV generations alone might not be sufficient to satisfy the powerquality requirements in the modern power system [1-6]. However, due to intermittent power generations from WP and PV, the unbalance of generation and load demand cause the large frequency fluctuation problem in the power system [3-4].

On the other hand, in the transport sector, Electric Vehicles (EV) has been developed as environmentally friendly vehicles. Other research groups has developed power system control methods by use of plenty of customers' appliances such as EV and heat pump water heaters (HPWH), as well as dispatching methods of the LFC signal [1-3]. These new appliances have energy storages such as battery or hot water tank. They are conceived as controllable loads because the power consumption can be changed during battery charging and discharging or water heating as long as the charging or heating is completed when the customer would like to use them. The HP and EV [4-6] are cooperated with TB and GOV of diesel generator. Nevertheless, various system operating conditions, unpredictable PV patterns, etc., cause system uncertainties Due to the system uncertainties such as system parameters variation, the conventional controllers which are designed without taking system uncertainties into account in the controller design, may lose the control effect and fail to damp the frequency deviation. This will affect the quality of supply and may deteriorate the system stability.

In fact, many control strategies have been proposed and investigated by several researchers for LFC design of power systems [1-6] with and without nonlinearities. This paper studies the effect of show the superior robustness and frequency control effect of the proposed coefficient diagram method in comparison with the conventional controllers on the system frequency response. Basically CDM is an algebraic approach applied to a polynomial loops in the parameter space such special diagram called coefficient diagram, which is used in smart power system to carry the necessary design information and as the criteria of good design [11]. The CDM is fairly new and not well-known, but its basic principle has been known in industry and in control community for more than 40 years with successful application in servo control, steel mill drive control, gas turbine control, and spacecraft attitude control [12]. In this paper, CDM controller design of smart power system utilizing HPWHs and EVs for the load frequency control has been presented. The parameters of the polynomials of CDM technique have been designed based on the dynamic model of the power system. The CDM technique law produces its optimal output derived from a quadratic cost function minimization based on the dynamic model of the smart power system as shown in Fig. 1. A comparison has been made between the CDM and the traditional integral controller confirming the superiority of the proposed CDM technique. The simulation results proved that the proposed controller can be applied successfully to the application of smart power system load frequency control with HPWHs and EV including the support of the variable solar power and variable load demand. A simple frequency analysis model used in the simulation is shown in fig.1. This paper is organized as follows. Section II describes the design of HPWH model III. Section Discusses about EV concept in detail. Section IV. Dynamics and the employed models of the proposed a smart power system with the PV-EV-HPWH as well as proposed CDM controller. Section III describes the General consideration about CDM and its Structure. Section IV describes the Variable solar power generation. Section $\mathrm{V}$ presents three configurations for case studies of the proposed smart Power system. Section VI analyzes time-domain simulated results 
of three studied cases of proposed controller with an isolated small Power system under various operated conditions. And finally Specific conclusions are drawn in Section VII.

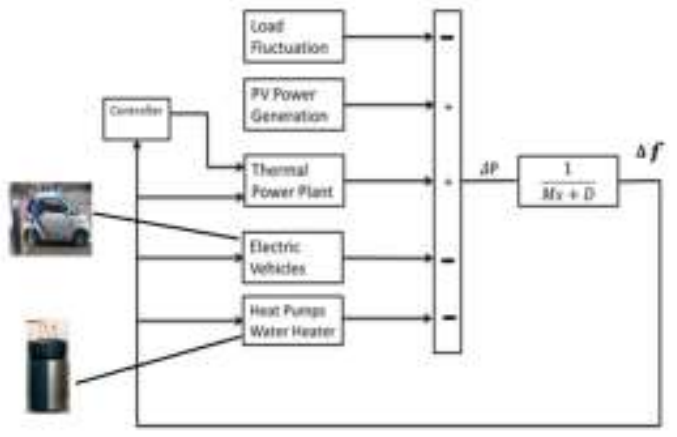

Fig. 1. Frequency Analysis Model

\section{HPWH model}

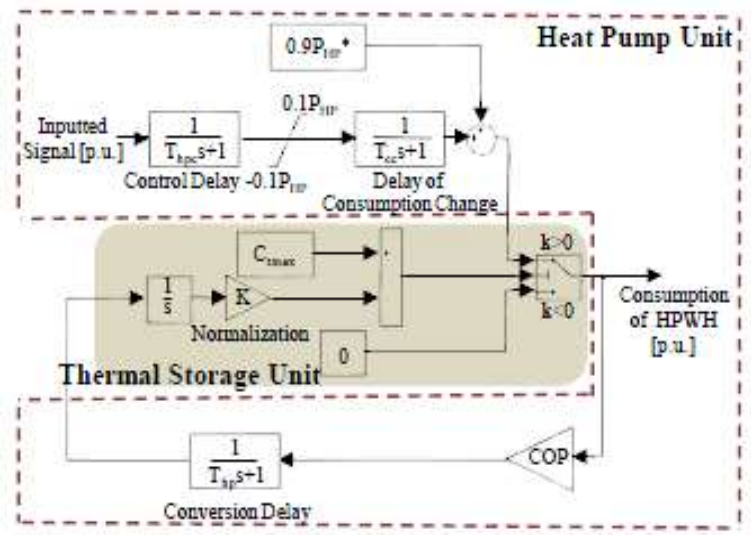

Fig. 2. Aggregated model of HPWH

An HPWH is a high-efficiency and energy-saving appliance [1], which has a tank to store hot water of the amount used in a day. The number of HPWHs in Japan is about 2 million in 2008 and it's expected to be 10 million in 2020 [1-3]. In general, an HPWH operates to heat water as much as the requested or estimated amount for the next day use during nighttime at low electricity price. It is assumed in this paper that the power consumption of the HPWHs can be controlled without decrease in efficiency in the range of $90 \% \pm 10$ of the rated power consumption according to the input control signal (LFC signal), that it is uncontrollable until the power consumption becomes stable $(0.25)[\mathrm{h}]$ after start), and that the operating period of the HPWHs also can be controlled [1-3].

In this Paper, we assumed the per unit rated power consumption according to the capacity of tank. The HPWH consists of starts unit and thermal unit as shown in fig. 2 . The parameters are shown in Table. 1. Given in [1-3]. The start unit is modeled for operation from zero to the steady state power consumption, which is approximate by first-order model with 60-second time delay on the basis that the power consumption of the air conditioner for home use becomes stable $0.25[\mathrm{~h}]$ after start. The thermal storage unit expresses the amount of total capacity of the hot water tank $\mathrm{C} \max [1]$. The input of the 
HPWH model id the Total control signals whereas the output is the total power consumption of the detail HPWH. In this paper it is assumed that there are 24.4 thousands HPWHs in the power system. The total power consumption can be calculated as:

\section{$24400 \times 1.22 \mathrm{kw} \times 0.2=100 \mathrm{MW}$}

\section{Table 1.}

Detail parameters of HPWH

\begin{tabular}{|c|c|}
\hline $\mathrm{T}_{h p c}$ Control Delay [sec] & 60 \\
\hline COP & 4 \\
\hline \multicolumn{2}{|l|}{$T_{\text {start }}$ Start Delay } \\
\hline Rated Power Consumption [kW] & 1.22 \\
\hline$T_{t h p} \quad$ Conversation Performance Delay [sec] & 600 \\
\hline$K_{N}$ Normalization Coefficient $[\mathrm{h} / \mathrm{s}]$ & $1 / 3600$ \\
\hline Total Thermal Storage Capacity [MWh] & 6,130 \\
\hline Total Power consumption [MW] & 100 \\
\hline \multicolumn{2}{|l|}{$T_{i g}$ Delay of Induction Motor [1] } \\
\hline Total controllable Capacity [MW] & \pm 10 \\
\hline
\end{tabular}

\section{EV model}

EVs which have electric motors instead of engines have gained much attention as the next generation vehicles. EVs can be controlled such as BESS in the grid because the batteries of EVs with two-way power converters can be charged and discharged corresponding to a LFC signal [4]. Our research model based on the concept of charging and discharging between the batteries of the EVs within the SOCs60\% to $90 \%$ and the power system in order to contribute to the power system operation and control. EVs discharge according to the LFC signal within the $90 \%$ SOCs and when it is charging state it give no response to the LFC signal. In Our system 60\% EVs is defined as controllable state and it can be discharge with LFC signal, Therefore EVs made to charge or discharge within the range of SOCs $60 \%$ to $90 \%$ with depend on the kwh capacity. It is assumed that the CLDC sends the control signal to the EVs and receives the information from the EVs via LFC signal. Within the range SOC $60 \%$ of the EVs, the User can plugged-in/out any time when the users like and should store the sufficient energy at plug-out for the next trip.

We assume that 3 thousand EVs are penetrated in an assumption area. Also, it is assumed that the SOC of each is synchronized, and EVs as a huge battery are introduced in frequency control. The discharge and charge power of EVs is limited by the number of EVs and SOCs. The power capacity of EVs is limited by the capacity of inverter and determined by the equation (2) and given in [6]

$$
E V k W=N E V \times C k W[k W]
$$

Where,

NEV: The number of EVs

CkW: The capacity of inverter 
The EV model is shown in Fig.3 and each parameter of EV shows in Table 2and given in [6]. We assume that EVs can charge and discharge with time lags 55000[sec] for input signal within the parameters of the charge and power capacity. When time reaches the EVs stops to charge or discharge.

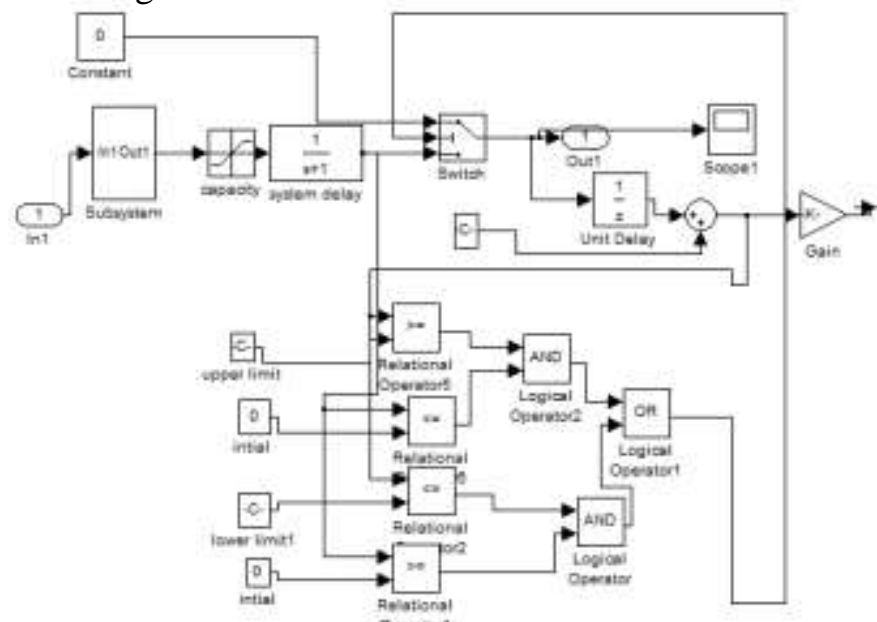

Fig. 3. EV Model

Table 2.

Parameters of EV

\begin{tabular}{|l|c|}
\hline Total battery capacity [p.u.MW] & $0.1125(90 \mathrm{MW})$ \\
\hline Power capacity [kW] & 3 \\
\hline Energy capacity [kWh] & 24 \\
\hline Initial SOC [\%] & 80 \\
\hline Upper limit of SOC[\%] & 90 \\
\hline Lower limit of SOC[\%] & 60 \\
\hline
\end{tabular}

\section{Variable solar power system}

The characteristic of PV system is illustrated in [3-4].Power output (in Watts) of a PV array which varies with irradiance and cell-surface temperature of a PV system is given by [3].The output power of the studied PV system is Determined byPpv= S $\phi \eta\{1-0.005(T a+25)\}$

The transfer function of PV can be given as:

$$
G_{p v}=\frac{K_{p v}}{T_{p v}+1}
$$

Where $\eta$ ranging from $9 \%$ to $12 \%$ is the conversion efficiency of the PV array, S $=4084$ $\mathrm{m} 2)$ is the measured area of the PV array, $\Phi(=1 \mathrm{~kW} / \mathrm{m} 2)$ is the solar radiation, and Ta is ambient Temperature in degree Celsius. The value of Ppv depends on Ta and $\Phi$ because $\eta$ and $\mathrm{S}$ are constant. In this paper, Ta is kept at $25^{\circ} \mathrm{C}$ and $\mathrm{Ppv}$ is linearly varied with $\Phi$ only.

$[\mathrm{MW}]$ is assumed by

$$
\sigma_{\text {load }}=0.6 \sqrt{P_{\text {load }}}
$$


Fluctuation period is 5 to $30[\mathrm{~min}]$ regarding load Frequency control. Original random fluctuation is generated from white noise block and eliminating Lower than 5 [min] and higher than 30 [min] components by Low-pass and high-pass filters respectively. It is multiplied by the standard deviation and base load capacity to calculate output fluctuation on the model. Load fluctuation is shown in Fig. 6.

\section{Simple load model}

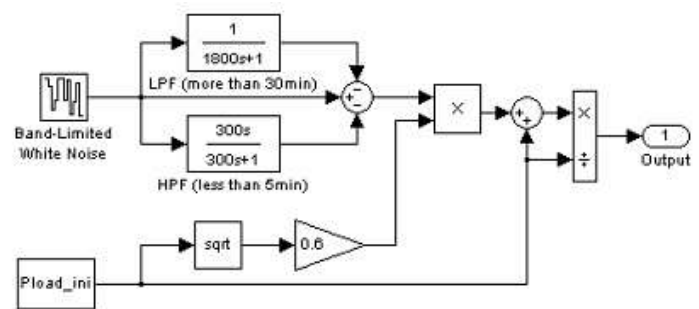

Fig. 4. Load model Load is defined in [9] in which standard deviation

\section{Overall system structure}

Figure 5.Illustratesthesmart power system, where PV is used as renewable energy source beside the thermal power.

In this section, a simplified frequency response model for a thermal power plant [1] and Photovoltaic is described [1]. A practical single area power system has the nominal parameters listed below in table.3. [14].

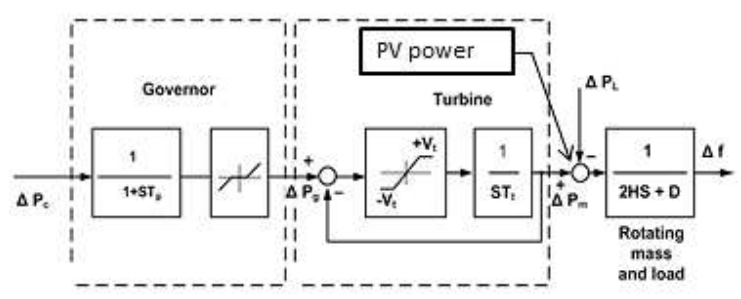

Fig. 5. Structure of the power system

Table 3.

Parameters and data of a practical single area power system

\begin{tabular}{|c|c|c|c|c|l|}
\hline $\mathbf{K}(\mathbf{s})$ & $\mathbf{D}(\mathbf{p u} / \mathbf{H z})$ & $\mathbf{H}(\mathbf{p u} . \mathbf{s e c})$ & $\mathbf{R}(\mathbf{H z} / \mathbf{p u})$ & $\mathbf{T}_{\mathbf{g}}(\mathbf{s e c})$ & $\mathbf{T}_{\mathbf{t}}(\mathbf{s e c})$ \\
\hline$-0.3 / \mathrm{s}$ & 0.015 & 0.08335 & 3.00 & 0.08 & 0.4 \\
\hline
\end{tabular}

The overall generator-load dynamic relationship between the supply error $\left(\Delta \mathbf{P}_{\mathbf{d}}-\Delta \mathbf{p}_{\mathbf{L}}^{\prime}\right)$ and the frequency deviation $(\Delta \mathbf{f})$ can be expressed as:

$$
\dot{\Delta} \mathbf{f}=\left(\frac{1}{M}\right) \cdot \Delta \mathbf{P}_{d}-\left(\frac{1}{M}\right) \cdot \Delta \mathbf{p}_{L}^{\prime}-\left(\frac{\mathbf{D}}{\mathbf{M}}\right) \cdot \Delta \mathbf{f}
$$

Where $\Delta \boldsymbol{p}_{L}^{\prime}=\boldsymbol{P}_{L}+\boldsymbol{P}_{E V}+\boldsymbol{P}_{H P}-\boldsymbol{P}_{P V}$

The dynamic of the diesel generator can be expressed as: $\Delta \dot{\mathbf{P}}_{\mathbf{d}}=\left(\frac{\mathbf{1}}{\mathbf{T}_{\mathbf{t}}}\right) \cdot \Delta \mathbf{P}_{\mathbf{g}}-\left(\frac{\mathbf{1}}{\mathbf{T}_{\mathbf{t}}}\right) \cdot \Delta \mathbf{P}_{\mathbf{d}}$

The dynamic of the governor can be expressed as: 


$$
\Delta \dot{\mathbf{P}}_{\mathrm{g}}=\left(\frac{1}{\mathrm{~T}_{\mathrm{g}}}\right) \cdot \Delta \mathrm{P}_{\mathrm{c}}-\left(\frac{1}{\mathrm{R} \cdot \mathrm{T}_{\mathrm{g}}}\right) \cdot \Delta \mathrm{f}-\left(\frac{1}{\mathrm{~T}_{\mathrm{g}}}\right) \cdot \Delta \mathrm{P}_{\mathrm{g}}
$$

\section{Coefficient diagram method}

In general, the classical control and modern control are mainly used in control design. Additionally there is a third approach generally called as algebraic design approach [12]. The Coefficient Diagram Method (CDM) is one of the algebraic design approaches, where the coefficient diagram is used instead of Bode diagram, and the sufficient condition for stability by Lipatov constitutes its theoretical basis [10].

The CDM is a technique to arrange the poles of a closed loop transfer function, in order to get wanted response in the time domain[11,13].According to Fig.5 the Coefficient Diagram provides to know the stability, time response and robustness characteristics of systems in a single diagram, which is important for systems with large characteristic polynomial degree. In coefficient diagram, logarithmic vertical axis shows the coefficients of characteristic polynomial $\left(\mathrm{a}_{\mathrm{i}}\right)$, stability indices $\left(\gamma_{\mathrm{i}}\right)$ and equivalent time constant $(\tau)$ whereas the horizontal axis shows the order i values corresponding to each coefficients. The degree of convexity obtained from coefficients of the characteristic polynomial gives a measure of stability, whereas the general inclination of the curve measures the speed of response and the shape of the $a_{i}$ curve due to plant parameter variation measures the robustness.

The block diagram of a single input single output (SISO) linear time invariant system with CDM control is shown in Fig.4, where

$\mathrm{N}(\mathrm{s})$ is numerator polynomial.

$\mathrm{D}(\mathrm{s})$ is denominator polynomial of the plant transfer function.

$\mathrm{A}(\mathrm{s})$ is considered as forward denominator polynomial.

$\mathrm{F}(\mathrm{s})$ and $\mathrm{B}(\mathrm{s})$ are considered as reference numerator and feedback numerator polynomials.

In the CDM controller, the transfer function of the controller has two numerators, which implies a 2DOF system structure. In this method $r$ is taken as the reference input to the system, $\mathrm{u}$ as the controller signal, $\mathrm{d}$ as the external disturbance signal and $\mathrm{y}$ is denoted as the output of the control system.

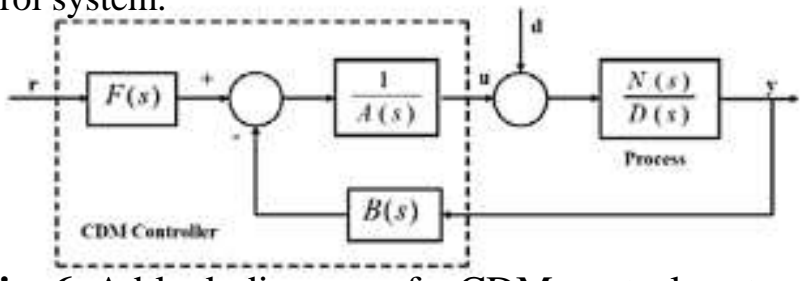

Fig. 6. A block diagram of a CDM control system.

$$
y=\frac{N(s) F(s)}{P(s)} r+\frac{A(s) N(s)}{P(s)} d
$$

Where $P(\mathrm{~s})$ is considered as the characteristic polynomial of the closed-loop system and is defined by

$$
P(s)=A(s) D(s)+B(s) N(s)
$$


$\mathrm{A}(\mathrm{s})$ and $\mathrm{B}(\mathrm{s})$ are considered as the control polynomial and is defined as

$$
A(s)=\sum_{i=0}^{P} l_{i} s^{i} \operatorname{and} B(s)=\sum_{i=0}^{q} k_{i} s^{i}
$$

For practical realization, the condition $\mathrm{p} \geq \mathrm{q}$ must be satisfied. To get the characteristic polynomial $\mathrm{P}(\mathrm{s})$, the controller polynomials from (6) are substituted in (5) and are given as

$$
P(s)=\sum_{i=0}^{P} l_{i} s^{i} D(s)+\sum_{i=0}^{q} k_{i} s^{i} N(s)=\sum_{i=0}^{n} a_{i} s^{i}, a_{i}>0
$$

CDM needs some design parameters with respect to the characteristic polynomial coefficients which are the equivalent time constant $(\tau)$ (which gives the speed of closed loop response), the stability indices $\left(\gamma_{i}\right)($ which give the stability and the shape of the time response), and the stability $\operatorname{limits}\left(\gamma_{i}{ }^{*}\right)$. The relations between these parameters and the coefficients of the characteristic polynomial $\left(a_{i}\right)$ can be described as follows:

$$
\begin{aligned}
& \gamma_{i}=\frac{a_{i}^{2}}{a_{i+i} a_{i-1}} \quad, i \in[1, n-1] \quad, \gamma_{0}=\gamma_{n}=\infty \\
& \tau=\frac{a_{1}}{a_{0}} \\
& \gamma_{i}^{*}=\frac{1}{\gamma_{i-1}}+\frac{1}{\gamma_{i+1}} \quad, i \in[1, n-1]
\end{aligned}
$$

According to Manabe's standard form, $\gamma_{i}$ values are selected as $\{2.5,2,2 \ldots 2\}$. The above $\gamma_{\mathrm{i}}$ values can be changed by the designer as per the requirement. Using the key parameters $\left(\tau\right.$ and $\left.\gamma_{i}\right)$, target characteristic polynomial, $P_{\text {target }}(\mathrm{s})$ can be framed as

$$
\begin{aligned}
& P_{\text {tareget }}=a_{\circ}\left[\left\{\sum_{i=2}^{n}\left(\prod_{j=1}^{i-1} \frac{1}{\gamma_{i-j}^{j}}\right)(\tau s)^{i}\right\}+\tau s+1\right] \\
& \text { Where } P(s)=P_{\text {target }}(s)
\end{aligned}
$$

Also, the reference numerator polynomials $\mathrm{F}(\mathrm{s})$ can be calculated from:

$$
F(s)=\left(\left.P(s)\right|_{s=0}\right) / N(s)
$$

The simulation studies are carried out for the proposed controller with generation rate constraint (GRC) of $10 \%$ p.u. per minute. The maximum value of dead band for governor is specified as $0.05 \%$. The parameters of the CDM controller are set as follows:

The time constant can be taken as 2 sec. , and from (11)

$$
P_{\text {tareget }}=1+2 S+1.6 S^{2}+0.64 S^{3}+0.128 S^{4}+0.0128 S^{5}
$$

The stability indices $\left(\gamma_{\mathrm{i}}\right)$ have been chosen as:

$$
\gamma_{i}=[2.5,2,1.25,5.12] \quad, i \in[1,4] \quad, \gamma_{0}=\gamma_{5}=\infty
$$

And the stability limits $\left(\gamma_{\mathrm{i}}{ }^{*}\right)$ are:

$$
\gamma_{i}^{*}=[0.5,1.2,0.6953,0.8] \quad, i \in[1,4]
$$

And choosing $\mathrm{k}_{0}=1$, then

$$
\begin{aligned}
& B_{i}=1+1.036 S+S^{2} \\
& A_{i}=.008+2.77 S+2.4 S^{2}
\end{aligned}
$$




\section{Result and discussion}

Computer simulations have been carried out in order to validate the effectiveness of the proposed scheme. The Mat lab/Simulink software package has been used for this purpose.

\subsection{First case}

The system performance with the proposed CDM controller in case of solar power generation variable load demand at nominal parameters is tested and compared with the conventional controller utilizing HPWHs and EV. Fig. 7 and Fig. 8show the participation of the Variable solar power fluctuation and variable load demand with respect of $20 \mathrm{~min}$ to 40min by using Low pass filter and high pass filter. In Fig. 4, the results from It has been noticed that with the proposed CDM controller with variable solar power generation and load demand, the system is more stable and fast compared to the system with conventional controllers utilizing controller load such as the HPWHs and EVs. The fig 9 depicts the discharging power of $\mathrm{EV}$ and power consumption of HP respectively.

\section{Effectiveness of the Proposed CDM with controllable loads (EV and HPWH)}

The simulation results of the frequency analysis are described in this subsection. Table 4 summarizes the max values of the frequency deviation in given $1.5[\mathrm{~h}] . \mathrm{In}$ the simulation conditions, the EVs and HPs are part of LFC with Integral and proposed CDM Controller respectively. The max value of the frequency deviation is used as an index to show the performance of controllers in the Load frequency control. Table 4 shows that the frequency control becomes more effective with proposed CDM controller for system including both the EVs and HPs

\section{Table 4.}

Max values of frequency deviation $[\mathrm{Hz}]$

\begin{tabular}{|c|c|}
\hline Conditions & Max Values [HZ] \\
\hline With HP and EV (Integral Controller) & 0.013 \\
\hline With HP and EV ( CDM Controller ) & 0.007 \\
\hline
\end{tabular}
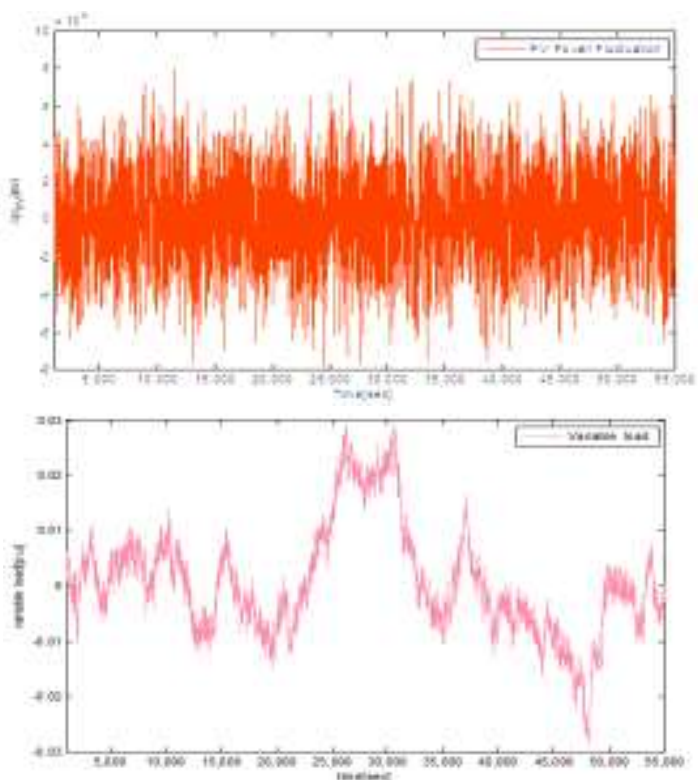

Fig. 7. variable solar Power and Variable Load demand respectively 

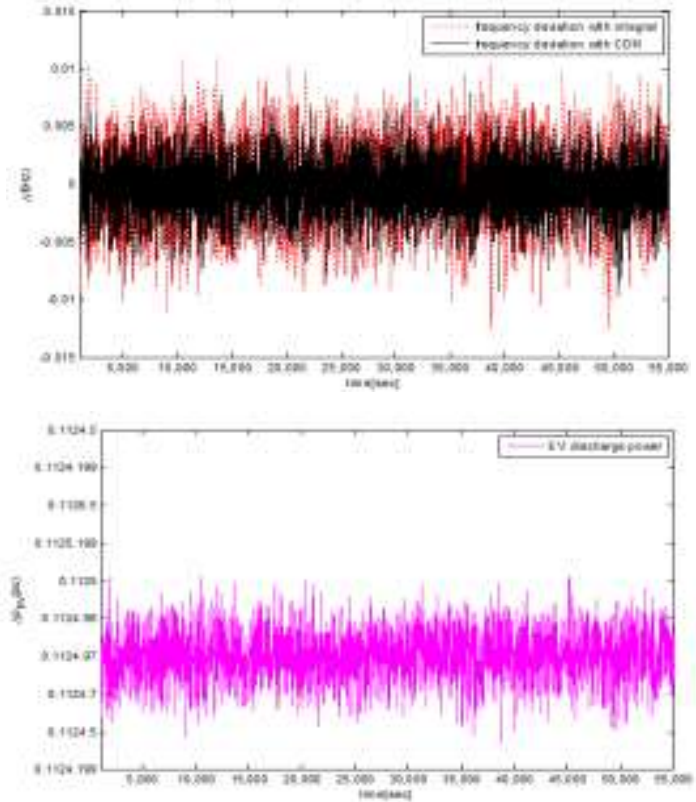

Fig. 8. Frequency deviation after utilizing HPWH and EV.(Solid line) with $\mathrm{CDM}$ and (dashed) with Integral controller

\subsection{Second case}

In this case the robustness of the proposed system against parameters uncertainty is validated. In this case, both of the governor and turbine time constants are increased to $T_{g}=0.14 \mathrm{sec}$ and $T_{d}$ $=7$ sec., respectively. Figure 10 depicts the system frequency response with proposed CDM and conventional Integral controller in this case of study. The random load change and PV power with HPWHs and EVs responses are assumed to be same as described in the first case. It has been shown that, with CDM controllers, the system response is more convenient.

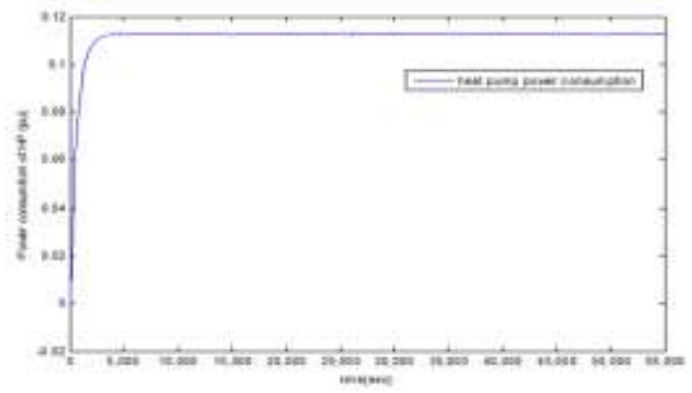

Fig. 9. EVs discharge Power responses and Power consumption responses from HP due to variable 


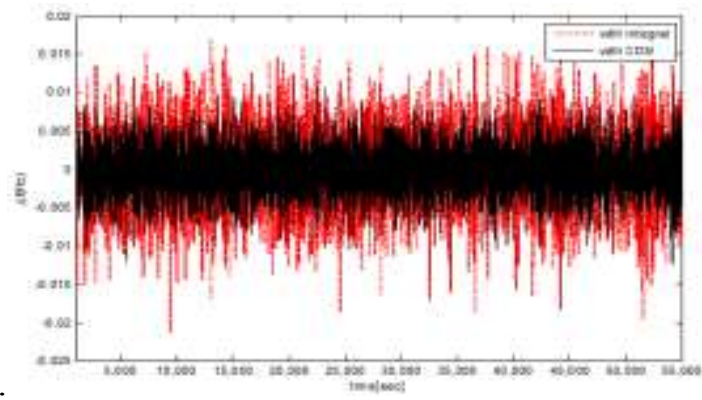

Fig. 10. Frequency deviations with parameter change case. (Solid line) with $\mathrm{CDM}$ and (dashed) with Integral controller.

\title{
9. Conclusion
}

This paper studies the merging of fluctuation of variable solar power utilizing HPs and EVs in a smart power system controlled by a robust load frequency control based on the coefficient Diagram method. Digital simulations have been carried out in order to validate the effectiveness of the proposed scheme. The proposed controller has been tested for mismatched generation. A performance comparison between the proposed CDM and conventional integrator controller in the presence of variable solar power fluctuation is carried out. The simulation results demonstrate that the closed-loop system with CDM controller is robust against the perturbation of the system and has desirable performance in comparison of classical integral control design in all of the performed test scenarios. Also, it was denoted that HPWHs and EVs has a positive effect on the total response of the smoothing effect of Variable solar power fluctuation in the smart Power system.

\section{Nomenclature}

$\Delta \mathrm{P}_{\mathrm{g}}$ : The governor output change $\Delta \mathrm{P}_{\mathrm{d}}$ : The thermal power change

$\Delta \mathrm{f}$ : The frequency deviation $\Delta \mathrm{P}_{\mathrm{L}}$ : the load change

$\Delta \mathrm{P}_{\mathrm{c}}$ : Supplementary control action $\Delta \mathrm{P}_{\mathrm{PV}}$ : the photovoltaic power change

$\Delta \mathrm{P}_{\mathrm{EV}}$ : charging power of plug-in hybrid electric vehicle $\Delta \mathrm{P}_{\mathrm{HP}}$ : Power consumption of heat pump.

$\mathrm{M}$ : Equivalent inertia constantD: equivalent damping coefficient

$\mathrm{R}$ : speed droops characteristic

$\mathrm{T}_{\mathrm{g}}$ : Governor time constant $\mathrm{T}_{\mathrm{d}}$ : turbine time constants.

$\mathrm{K}(\mathrm{s})$ : Conventional Integral Controller

\author{
Abbreviations or Subscripts \\ EV: Electric vehicle. \\ CDM: Coefficient diagram method \\ HPWH: Heat Pump Water Heater \\ SOC: State Of Charge \\ CLDC: Control Load Dispatching Centre
}

\section{REFERENCES}

[1] Taisuke Masuta*, Akihiko Yokoyama,Yasuyuki Tada, "A Supplementry Load Frequency Control Method By Use Of Heat Pump Water Heaters On Customers Side Without Realtime",17th Power Systems Computation Conference, StockholmSweden - August 22-26, 2011

[2] Taisuke Masuta, Akihiko Yokoyama, Member, IEEE, and Yasuyuki Tada, Member, IEEE,"System Frequency Control by Heat Pump Water Heaters (HPWHs) on Customer 
Side Based onStatistical HPWH Model in Power system witha Large Penetration of Renewable EnergySources",2010 International Conference on Power System Technology.

[3] Taisuke Masuta, Akihiko Yokoyama, Member, IEEE, and Yasuyuki Tada, Member, IEEE,"Modeling of a Number of Heat Pump WaterHeaters as Control Equipment for LoadFrequency Control in Power Systems",at the 2011 IEEE Trondheim PowerTech.

[4] Koichiro Shimizu*, Taisuke Masuta, Yutaka Ota, and Akihiko Yokoyama, "A New Load Frequency Control Method In Power System Using Vechicles-To-Grid System Considering Users's Convenience " confenerce, stockholm Sweden- August 22-26,2011

[5] Koichiro Shimizu,Taisuke Masuta, Yuyaka Ota, Member, IEEE, and Akihiko Yokoyama, Member,IEEE,"Load Frequency Control in Power SystemUsing Vehicle-to-Grid System Considering theCustomer Convenience of Electric Vehicles",2010 International Conference on Power System Technology.

[6] T. Shimamura, D. Yamashita, K. Koyanagi, Y. Nakanishi and R. Yokoyama,"Evaluation of Smoothing Effect of Wind Power Generator Aggregationon Power System Operation",International Conference on Renewable Energies and Power Quality (ICREPQ'13).Bilbao (Spain), 20th to 22th March, 2013.

[7] Dong-Jing Lee and Li Wang, Senior Member, IEEE,"Small-Signal Stability Analysis of an AutonomousHybrid Renewable Energy Power Generation/EnergyStorage System Part I: Time-Domain Simulations",Manuscript received November 7, 2006; revised March 31, 2007. Paper no.TEC-00522-2006.

[8] Tomonobu Senjyu1, Manoj Datta1, Atsushi Yona1, Toshihisa Funabashi2, and Chul-Hwan Kim, "A Minimal-Order Observer Based Control Method for PV Generator to Reduce Frequency Deviations of Power System", Power and Energy Conference, 2008. PECon 2008. IEEE 2nd International.

[9] T.Michigami, Senior Member, IEEE, and T.Ishi N on Member, IEEE, "Construction of Fluctuation Load Model and Dynamic Simulation with LFC Control of DC Power System and Frequency Converter Interconnection",0-7803-7525-4/02/\$17.00 02002 IEEE.

[10] Shunji Manabe, "Importance of Coefficient Diagram in Polynomial Method", Proceedings of the 42nd IEEE Conference on Decision and Control Maui, Hawaii USA, December 2003

[11] S. Manabe, "Coefficient Diagram Method", 14th IFAC Symp on Automatic Control in Aerospace, Seoul, 1998

[12] Rinu raj R. R, and L. D. Vijay Anand "Design and Implementation of a CDM-PI Controller for a Spherical Tank Level System", International Journal on Theoretical and Applied Research in Mechanical Engineering (IJTARME), Volume-2, Issue-1, 2013.

[13] S. Manabe, "A Low Cost Inverted Pendulum System For Control System Education", The 3rd IFAC Symposium on advances in Control Education, Tokyo, 1994

[14] H. Bevrani, "Robust Power System Control", Springer, New York, pp. 15-61, 2009. 